\title{
UNRESECTABLE HEPATOBLASTOMA, LIVING DONATION AND PRE-TRANSPLANT FACTORS ASSOCIATED WITH EVENT-FREE SURVIVAL
}

\author{
Joao Seda-Neto ${ }^{1}$, Flávia Feier ${ }^{2}$, Renata Pugliese ${ }^{3}$, Helry Candido ${ }^{2}$, Rodrigo Vincenzi ${ }^{2}$, \\ Gilda Porta ${ }^{3}$, Irene Miura ${ }^{3}$, Vera Baggio ${ }^{2}$, Adriana Porta ${ }^{3}$, Celso Rodrigues ${ }^{2}$, Cecilia da \\ Costa $^{4}$, Renato Melaragno ${ }^{5}$, Sidnei Epelman ${ }^{6}$, Ana Paula Winneschhofer ${ }^{7}$, Eduardo \\ Fonseca $^{2}$, and Paulo Chapchap ${ }^{2}$ \\ ${ }^{1}$ Hospital Sirio-Libanes \\ ${ }^{2}$ Hospital Sirio Libanes \\ ${ }^{3}$ Hospital Sirio Libanes/ Hospital AC Camargo \\ ${ }^{4}$ Fundação Antônio Prudente \\ ${ }^{5}$ Hospital do Câncer AC Camargo \\ ${ }^{6}$ Hospital Santa Marcelina de Sao Paulo, Brazil \\ ${ }^{7}$ Hospital Infantil Joana de Gusmao
}

March 22, 2021

\begin{abstract}
Background: Hepatoblastoma (HB) treatment has improved over time with established chemotherapy (Qtx) protocols, and liver resection or liver transplantation (LT). However, the right timing for LT and adequate patient selection are key to achieve acceptable disease-free survival rates in patients with unresectable HB. Few groups have reported such factors in the setting of living donor liver transplantation (LDLT). Procedure: This single-center retrospective analysis of 28 children with HB submitted to LDLT aimed at determining the pre-transplant factors associated with worse post-transplant event-free survival. Results: Patients were divided in groups according to the occurrence of the event (recurrence/death) after LDLT - 10 patients in the event-yes and 18 patients in the event-no. Probability of 5-y event-free survival was $63.9 \%$. Alpha-fetoprotein (AFP) reduction post-Qtx $>70 \%$ had a good performance for the occurrence of the event, with a calculated AUC of 0.8 . A scoring system was derived from the pre-transplant risk factors (AFP reduction $<70 \%$, time from diagnosis to LDLT $>12$ months, rescue LT) for the probability of the event: no risk factor present (15.4\%), one risk factor present (33.3\%), and $>2$ risk factors present (66.7\%), ( $\mathrm{p}=0.02)$. Conclusion: LDLT for HB is the preferred treatment option for unresectable HB, with no distant metastasis and adequate response to Qtx. The pre-transplant factors composing the risk score should be critically evaluated in order to move forward with the LDLT. However, due to the limited number of patients in this study, a larger number of patients is required to corroborate these findings.
\end{abstract}

\section{INTRODUCTION}

Hepatoblastoma (HB) is the most common primary hepatic neoplasm in children. The mainstays of HB treatment are surgical resection and cisplatin-doxorubicin (PLADO) chemotherapy (Qtx). If the tumor is considered unresectable at diagnosis, neoadjuvant chemotherapy can make the lesion resectable in up to $80 \%$ of the patients ${ }^{1}$. For patients with PRETEXT III or IV that remain unresectable after neoadjuvant Qtx, and do not present distant metastasis, liver transplantation (LT) is the preferred treatment alternative. Current 10-year post-LT overall survival for unresectable HB is over $80 \%^{2}$. 
Although patients with HB receive exception points on the waiting list to compete with other children with end-stage liver disease for a liver graft, the timing to perform the LT is crucial for a better outcome. Usually these patients have an optimal treatment window - after completion of the Qtx - when the transplant can be performed. The importance of living donor liver transplantation (LDLT) in this context has been previously described $^{3,4}$. The majority of the reports on LDLT for HB are based on a limited number of cases, and most studies show recurrence rates from $20 \%$ to $37.5 \%^{4-6}$.

It is well known that response to chemotherapy, manifested as either a decrease in tumor size or a significant decrease in alpha-fetoprotein (AFP) level, is the most important prognostic factor for successful LT ${ }^{7}$. However, lengthy courses of preoperative chemotherapy while the tumor remains unresectable should be avoided due to diminishing effects on the tumor, combined with the substantial risk of inducing Qtx resistance with the prolonged exposure ${ }^{8}$.

The ideal scenario when treating patients with unresectable HB should include response to Qtx and the availability of liver graft for transplantation. However, how far to push the transplant indication in patients with a marginal response to chemotherapy (but no distant metastasis) and with an available living donor? In the present study, the outcome of 28 patients who received LDLT is reported with emphasis in the factors associated with recurrence/death after transplantation.

\section{METHODS}

A total of 28 primary pediatric LDLT for HB were performed in patients under 18 years of age at Hospital Sírio-Libanês and A. C. Camargo Cancer Center, São Paulo, Brazil, from 1996 to 2019. The clinical variables collected were gender, age, PELD score (Pediatric End-Stage Liver Disease scoring system), type of neoadjuvant Qtx (PLADO versus other regimens), pre- and post-Qtx AFP levels, \%AFP reduction post Qtx (AFP pre-Qtx - AFP post-Qtx/AFP pre-Qtx), PRETEXT stage, primary versus rescue LDLT, time between diagnosis and LDLT, presence of metastases at diagnosis, follow-up time. These variables were used for determining the factors associated with event-free and overall survival in this cohort. An event was defined as HB recurrence or patient death, whichever came first. The patients were then divided in two groups: Event-No and Event-Yes. Rescue transplant was defined as transplant after a previous liver resection for HB.

Pulmonary metastasis at the time of diagnosis was not considered a contra-indication for transplantation as long as it was treated either with surgery or achieved complete remission after chemotherapy.

The same medical team was responsible for the clinical and surgical transplantation procedures in both hospitals. All patients were referred to our center by different pediatric oncology centers in the country and had received pre-transplant chemotherapy regimen as determined by each center. In this retrospective study, data was acquired through review of medical records and from a prospectively collected database. The hospitals' ethics committee approved this study. Vascular invasion was studied only in liver explant analysis.

Post-operative care

Patients with platelet count $>50,000 / \mathrm{mm}^{3}$ were kept on dipyridamole $(1 \mathrm{mg} / \mathrm{kg} /$ day $)$ or aspirin $(3 \mathrm{mg} / \mathrm{kg})$ for 3 months after the transplant. Tacrolimus (FK 506, Prograf) and steroids were used for immunosuppression. Details of post-operative clinical management have been previously described and were equal to the care provided for children transplanted for other reasons (metabolic diseases and cirrhosis) ${ }^{9,10}$.

Statistical analysis

Continuous variables were tested for normality with the Kolmogorov-Smirnov and Shapiro Wilk tests. The values are expressed either as mean \pm standard deviation $(\mathrm{SD})$ or median, and 25 and 75 percentiles. The categorical data are presented as absolute values and percentages and were tested using Pearson $\chi^{2}$ test and Fischer's Exact Test. The comparison between groups was performed using the Kruskal Wallis test or Pearson $\chi 2$ test and Fischer's Exact Test, as appropriate. Event-free survival and patient survival analysis was conducted according to the Kaplan-Meier product-limit estimates, and patient subgroups were compared using a two-sided log-rank test. 
A Cox-Regression analysis was conducted to evaluate the association of each clinically or statistically significant $(\mathrm{p}<0.1)$ variable with the outcome (event-free survival).

A scoring system was derived from the Cox-Regression analysis to predict the outcome probability (recurrence/death) according to the number of factors presented by the patient in the pre-transplant period.

The area under the receiver operating characteristic curve (AUC ROC) was the tool used to evaluate the association of AFP reduction with the outcome (event-free survival). Therefore, AUC ROC ranges from 0 to 1 , with 1 corresponding to a perfect score. AUC ROC [?] 0.7 are considered to have very little predictive ability. The level of statistical significance adopted was P [?] 0.05. All analyses were performed using the SPSS 21.0 statistical package (IBM, Inc., Chicago, IL, USA).

\section{RESULTS}

Twenty-eight patients underwent LDLT for HB during the study period. A total of 21 (75\%) patients were classified as PRETEXT IV, $4(14.3 \%)$ patients as PRETEXT III and $3(10.7 \%)$ patients were rescue LT. All of them received pre-LT Qtx: $13(46.4 \%)$ received PLADO, $10(35.8 \%)$ received alternative regimens, and for $5(17.8 \%)$ patients the Qtx regimen was not informed. Eighteen patients were event-free, and were included in the Event-No group. A total of 10 patients experienced an event (recurrence or death), and were included in the Event-Yes group (Table 1).

Comparing the two groups, the statistically different pre-LDLT variables were: time from diagnosis to the LDLT $>12$ months $(\mathrm{p}=0.09)$, post-Qtx AFP levels $(\mathrm{p}=0.07)$, and AFP post-Qtx reduction $>70 \%(\mathrm{p}=0.05)$. The only statistically different post-LDLT variable among groups was the presence of vascular invasion in the explant analysis $(\mathrm{p}=0.002)$ (Table 1$)$. Mean hospital stay was of $13.5+7.8$ days in the Event-no group vs $12.5+4.6$ days in the Event-yes group $(\mathrm{p}=0.96)$.

Post-LDLT overall 5-y survival probability was 66\% (Figure 1A) and 5-y event-free survival probability was $63.9 \%$ (Figure 1B). In those patients who achieved an AFP reduction post-Qtx [?] 70\%, 5-y event-free survival was of $81.3 \%$ vs. $40 \%$ in those who did not $(\mathrm{p}=0.02)$ (Figure $2 \mathrm{~A})$. Patients in which the time from HB diagnosis to LDLT was> 12 months had a 5-y event-free survival rate of $40 \%$ vs $77 \%$ for those who performed the LDLT in $<12$ months from the diagnosis $(\mathrm{p}=0.01$; Figure $2 \mathrm{~B})$. Patients who received the PLADO pre-LDLT Qtx regimen (high-risk SIOPEL) had a 5-y event-free survival of $76.9 \%$ versus $50 \%$ for those who received other Qtx regimens $(\mathrm{p}=0.14)$ (Figure $2 \mathrm{C}$ ). The 5-y event-free survival in primary LDLT was $67.7 \%$ versus $33.3 \%$ for those who underwent rescue $\mathrm{LT}(\mathrm{p}=0.04)$ (Figure $2 \mathrm{D})$. In the liver explant analysis, the presence of vascular invasion also impacted in the 5-y event-free survival: no vascular invasion $(82.4 \%)$ vs microvascular invasion $(50 \%)$ vs macrovascular invasion $(20 \%)(\mathrm{p}=0.04)$.

In order to evaluate the performance of the test (AFP reduction [?]70\%) with the occurrence of the event (recurrence/death), an ROC curve analysis was made, and the calculated AUC was of 0.8 (Figure 3).

The pre-LDLT factors identified that were associated with the occurrence of the event (recurrence/death) underwent a Cox-Regression analysis. The hazard-ratios (HR) were calculated in order to create a scoring system (Figure 4). Each risk factor (AFP reduction $<70 \%$, time from diagnosis to LDLT $>12$ months, rescue LT) represents 1 point in the pre-LDLT scoring system to predict the probability of the event: no risk factor present $(15.4 \%)$, one risk factor present $(33.3 \%)$ and $>2$ risk factors present $(66.7 \%), \mathrm{p}=0.02$ (Figure $5)$.

\section{DISCUSSION}

The outcome of patients with HB changed dramatically after the establishment of Qtx protocols associated with liver resection, pioneered by the SIOPEL ${ }^{11}$ and $\mathrm{COG}^{12}$ study groups. The 10-y expected overall survival with primary LT was $85 \%$, and $40 \%$ in patients who underwent LT as a "rescue therapy" 13 in the SIOPEL-1 study. After the initial studies, and others that followed, the absolute indication for LT, and the basis for considering a tumor unresectable after neoadjuvant Qtx, is POST-TEXT IV or POST-TEXT III with major 
vascular tumor involvement ${ }^{14,15}$. Five-year patient survival in the present report is similar to event-free survival, since recurrence meant fatality in almost all patients.

Determining the risk factors associated with patient survival is paramount to an improved selection of patients for LT, but efforts to identify such prognostic factors in the setting of a rare tumor have been hampered by extensive fragmentation resulting in relatively small patient cohorts ${ }^{16}$. In the report of the Children's Hepatic tumors International Collaboration (CHIC), advanced PRETEXT group, macrovascular venous or portal involvement, contiguous extrahepatic disease, primary tumor multifocality and tumor rupture at enrollment, higher age ( $>8$ years), low AFP $(<100 \mathrm{ng} / \mathrm{ml})$, and metastatic disease were associated with the worst outcome; however, patients in all stages of HB were included.

Studies in children with HB undergoing LT, despite the limited number of patients enrolled in each cohort, seem to point to the same direction. Umeda et al. ${ }^{6}$, in a retrospective analysis of 24 children with unresectable HB submitted to LT, showed that the response to Qtx at LT, evaluated by the decline (>95\%) in serum AFP levels, could predict post-LT relapse for patients receiving both primary and rescue LT. Browne et al. ${ }^{17}$, in a cohort with 14 patients, demonstrated that a drop of $99 \%$ in peak AFP levels was associated with $100 \%$ survival following LT. Other authors also have correlated the trends in AFP levels ${ }^{18}$ or pretransplant AFP values with outcome ${ }^{2,19}$. In our study, a drop [?]70\% in AFP levels was associated with improved survival at 5 -years $(81.3 \%$ versus $40 \%)$. It showed that it is still safe to indicate LT for patients with a more modest, but still significant drop in AFP levels post-Qtx. The ROC curve analysis (Figure 3) presented an AUC of 0.8, demonstrating a good performance/correlation between the studied variable and event-free (event-No group) outcome.

Salvage or rescue LT has been associated with tumor recurrence and worse patient survival since the SIOPEL1 report $^{13}$. Many other studies replicated those results ${ }^{7,17,20-22}$, and the present study corroborates worst patient survival with rescue transplantation, where all primary tumors were assumed to be resected during the first operation. Time between diagnosis and LT $>12$ months was also associated with decreased patient survival (Figure 2b, $77 \%<12$ mo. vs. $40 \%>12$ mo., $\mathrm{P}=0.01$ ). Indeed, lengthier Qtx regimens are often required in high-risk subgroups, and are characterized by marked chemoresistance and poor outcome ${ }^{23,24}$.

The Cox-Regression analysis and the respective hazard ratios of the three variables (AFP reduction $<70 \%$, time from diagnosis to treatment [?] 12 months, and rescue LT) associated with increased risk of tumor recurrence/death in this series are shown in Figure 4. Placed together, the observed event-free survival was $84.6 \%$ (no factors), $66.7 \%$ (1 risk factor), and 32.3\% ([?] 2 risk factors). Most importantly, these are pre-operative risk factors that may be able to help families and physicians during the decision-making process, especially in the context of live donation. The advantage of timely LT must be weighted at all times against the risks of the surgical procedure in the living donor and the chances of cure of the children with unresectable HB. In our own experience, Candido et al. ${ }^{25}$ reported a rate of post-operative complication of $4.8 \%$ (29/601) in left liver segment donors used in pediatric LDLT with no patient mortality; however, the estimated rate of donor death "definitely" related to donor surgery has been reported to be $0.15 \%{ }^{26}$.

Vascular invasion has been associated with increased risk of recurrence ${ }^{27-29}$, and the explant analysis with presence of vascular invasion in the present study was also associated with recurrence/death [patient survival with no vascular invasion (82.4\%) vs. microvascular invasion (50\%) vs. macrovascular invasion $(20 \%)$ $(\mathrm{p}=0.04)]$. Most probably, if vascular invasion is detected in the pre-transplant staging (POSTTEXT), it might be useful to include this variable so as to compose a risk stratification to be used with the ones described in this publication; however, the present retrospective analysis did not allow such inclusion/comparison.

The present report shows the experience of a transplant center. The majority of the patients were referred to us from different oncology groups in the country, after the pretransplant chemotherapy regimens had already been defined. Despite the limitation in sample size, one should keep in mind that the study was designed to determine the risk factors for recurrence in a rare disease scenario, over a relatively long period of time. Also, chemotherapy regimens have changed over the study period, even the one used in the PLADO group. The authors recognize these shortcomings but they are inherent of the retrospective nature of this series. 
In conclusion, LDLT for HB is a treatment option for unresectable HB, with no distant metastasis and adequate response to Qtx. The following pre-transplant factors - AFP reduction $<70 \%$, time from diagnosis to LT > 12 months and rescue LT - were associated with higher recurrence/death risk and should be critically evaluated and discussed with the patient's family and the multidisciplinary team in order to move forward with the LDLT in a timely fashion in order to avoid drug toxicity and chemoresistance. However, due to the limited number of patients enrolled in this study, a larger number of patients is required to corroborate these findings.

\section{Conflicts of interest statement}

There was no external funding for this work. The authors do not have any commercial or financial connections that would pose a conflict of interest to the findings of this manuscript.

\section{REFERENCES}

1. Pritchard J, Brown J, Shafford E, et al. Cisplatin, doxorubicin, and delayed surgery for childhood hepatoblastoma: a successful approach-results of the first prospective study of the International Society of Pediatric Oncology.J Clin Oncol . Nov 15 2000;18(22):3819-28. doi:10.1200/JCO.2000.18.22.3819

2. Ramos-Gonzalez G, LaQuaglia M, O'Neill AF, et al. Long-term outcomes of liver transplantation for hepatoblastoma: A single-center 14-year experience. Pediatr Transplant. Jun 11 2018:e13250. doi: $10.1111 /$ petr. 13250

3. Kasahara M, Ueda M, Haga $\mathrm{H}$, et al. Living-donor liver transplantation for hepatoblastoma. Am $J$ Transplant . Sep 2005;5(9):2229-35. doi:10.1111/j.1600-6143.2005.01003.x

4. Sakamoto S, Kasahara M, Mizuta K, et al. Nationwide survey of the outcomes of living donor liver transplantation for hepatoblastoma in Japan. Liver Transpl . Mar 2014;20(3):333-46. doi:10.1002/lt.23803

5. Kueht M, Thompson P, Rana A, Cotton R, O'Mahony C, Goss J. Effects of an early referral system on liver transplantation for hepatoblastoma at Texas Children's Hospital.Pediatr Transplant. Jun 2016;20(4):515-22. doi:10.1111/petr.12699

6. Umeda K, Okajima H, Kawaguchi K, et al. Prognostic and therapeutic factors influencing the clinical outcome of hepatoblastoma after liver transplantation: A single-institute experience. Pediatr Transplant . Mar 2018;22(2)doi:10.1111/petr.13113

7. Pimpalwar AP, Sharif K, Ramani P, et al. Strategy for hepatoblastoma management: Transplant versus nontransplant surgery. J Pediatr Surg . Feb 2002;37(2):240-5. doi:10.1053/jpsu.2002.30264

8. Meyers RL, Tiao G, de Ville de Goyet J, Superina R, Aronson DC. Hepatoblastoma state of the art: pre-treatment extent of disease, surgical resection guidelines and the role of liver transplantation. Curr Opin Pediatr . Feb 2014;26(1):29-36. doi:10.1097/MOP.0000000000000042

9. Neto JS, Pugliese R, Fonseca EA, et al. Four hundred thirty consecutive pediatric living donor liver transplants: variables associated with posttransplant patient and graft survival. Liver Transpl . May 2012;18(5):577-84. doi:10.1002/lt.23393

10. Neto JS, Carone E, Pugliese V, et al. Living donor liver transplantation for children in Brazil weighing less than 10 kilograms. Liver Transpl . Aug 2007;13(8):1153-8. doi:10.1002/lt.21206

11. Brown J, Perilongo G, Shafford E, et al. Pretreatment prognostic factors for children with hepatoblastoma- results from the International Society of Paediatric Oncology (SIOP) study SIOPEL 1. Eur J Cancer . Jul 2000;36(11):1418-25. doi:10.1016/s0959-8049(00)00074-5

12. Malogolowkin MH, Katzenstein HM, Krailo M, Meyers RL. Treatment of hepatoblastoma: the North American cooperative group experience. Front Biosci (Elite Ed) . Jan 1 2012;4:1717-23. doi:10.2741/492 
13. Otte JB, Pritchard J, Aronson DC, et al. Liver transplantation for hepatoblastoma: results from the International Society of Pediatric Oncology (SIOP) study SIOPEL-1 and review of the world experience. Pediatr Blood Cancer. Jan 2004;42(1):74-83. doi:10.1002/pbc.10376

14. Meyers RL, Czauderna P, Otte JB. Surgical treatment of hepatoblastoma. Pediatr Blood Cancer . Nov 2012;59(5):800-8. doi:10.1002/pbc.24220

15. Otte JB. Progress in the surgical treatment of malignant liver tumors in children. Cancer Treat Rev . Jun 2010;36(4):360-71. doi:10.1016/j.ctrv.2010.02.013

16. Czauderna P, Haeberle B, Hiyama E, et al. The Children's Hepatic tumors International Collaboration (CHIC): Novel global rare tumor database yields new prognostic factors in hepatoblastoma and becomes a research model. Eur J Cancer . Jan 2016;52:92-101. doi:10.1016/j.ejca.2015.09.023

17. Browne M, Sher D, Grant D, et al. Survival after liver transplantation for hepatoblastoma: a 2-center experience. J Pediatr Surg . Nov 2008;43(11):1973-81. doi:10.1016/j.jpedsurg.2008.05.031

18. Isono K, Ohya Y, Lee KJ, et al. Pretransplant trends in alpha-fetoprotein levels as a predictor of recurrence after living donor liver transplantation for unresectable hepatoblastoma: A single-institution experience. Pediatr Transplant . Aug 2018;22(5):e13221. doi:10.1111/petr.13221

19. Lauferman L, Halac E, Aredes D, et al. Prognostic factors for event-free survival in liver transplantation for hepatoblastoma: A single-center experience.Pediatr Transplant . Dec 2019;23(8):e13581. doi:10.1111/petr.13581

20. Avila LF, Encinas JL, Leal N, et al. [Liver transplatation for malignant tumors in children]. Cir Pediatr . Oct 2007;20(4):189-93. Trasplante hepatico en tumores malignos en el nino.

21. Casas-Melley AT, Malatack J, Consolini D, et al. Successful liver transplant for unresectable hepatoblastoma. J Pediatr Surg . Jan 2007;42(1):184-7. doi:10.1016/j.jpedsurg.2006.09.017

22. Otte JB, de Ville de Goyet J. The contribution of transplantation to the treatment of liver tumors in children. Semin Pediatr Surg . Nov 2005;14(4):233-8. doi:10.1053/j.sempedsurg.2005.06.006

23. De Ioris M, Brugieres L, Zimmermann A, et al. Hepatoblastoma with a low serum alphafetoprotein level at diagnosis: the SIOPEL group experience. Eur J Cancer. Mar 2008;44(4):545-50. doi:10.1016/j.ejca.2007.11.022

24. Marin JJG, Cives-Losada C, Asensio M, Lozano E, Briz O, Macias RIR. Mechanisms of Anticancer Drug Resistance in Hepatoblastoma. Cancers (Basel) . Mar 22 2019;11(3)doi:10.3390/cancers11030407

25. Candido HL, da Fonseca EA, Feier FH, et al. Risk Factors Associated with Increased Morbidity in Living Liver Donation. J Transplant . 2015;2015:949674. doi:10.1155/2015/949674

26. Trotter JF, Adam R, Lo CM, Kenison J. Documented deaths of hepatic lobe donors for living donor liver transplantation. Liver Transpl . Oct 2006;12(10):1485-8. doi:10.1002/lt.20875

27. Reyes JD, Carr B, Dvorchik I, et al. Liver transplantation and chemotherapy for hepatoblastoma and hepatocellular cancer in childhood and adolescence. J Pediatr. Jun 2000;136(6):795-804.

28. von Schweinitz D, Wischmeyer P, Leuschner I, et al. Clinico-pathological criteria with prognostic relevance in hepatoblastoma. Eur J Cancer . 1994;30A(8):1052-8. doi:10.1016/0959-8049(94)90454-5

29. Okur MH, Yankol Y, Cimsit B, et al. Liver Transplant in Children with Hepatoblastoma. Exp Clin Transplant . Oct 2019;17(5):644-647. doi:10.6002/ect.2016.0110

\section{FIGURE LEGENDS}

Figure 1 
Kaplan-Meier product-limit estimates. Five-year patient survival probability (A), and 5-y event-free survival probability (B).

\section{Figure 2}

Kaplan-Meier product-limit estimates / 5-y event-free survival: (A) [?] 70\% reduction in AFP levels (81.3\%) vs. $<70 \%$ reduction $(40 \%), \mathrm{P}=0.02$, (B) Time from HB diagnosis to LDLT, $\mathrm{t}>12$ mo. (40\%) vs. $\mathrm{t}<12$ mo $(77 \%), \mathrm{P}=0.01,(\mathrm{C})$ PLADO Qtx regimen $(76.9 \%)$ vs. other Qtx regimens $(50 \%), \mathrm{P}=0.14$, (D) primary LDLT $(67.7 \%)$ vs. rescue LT (33.3\%), $\mathrm{P}=0.04$.

\section{Figure 3}

ROC curve analysis: performance AFP reduction [?] 70\% with recurrence/death. The calculated AUC was 0.8 .

\section{Figure 4}

Cox-Regression analysis showing the respective hazard-ratios for recurrence/death of the selected pretransplant variables.

\section{Figure 5}

Risk factors and event prediction probability. No-risk factor present (15.4\%), one risk factor present (33.3\%) and $>2$ risk factors present $(66.7 \%), \mathrm{P}=0.02$. Risk factors: rescue LT, $>12$ months from diagnosis to LT, and $<70 \%$ AFP reduction post-Qtx.

Figure 1.

A. Patient Survival

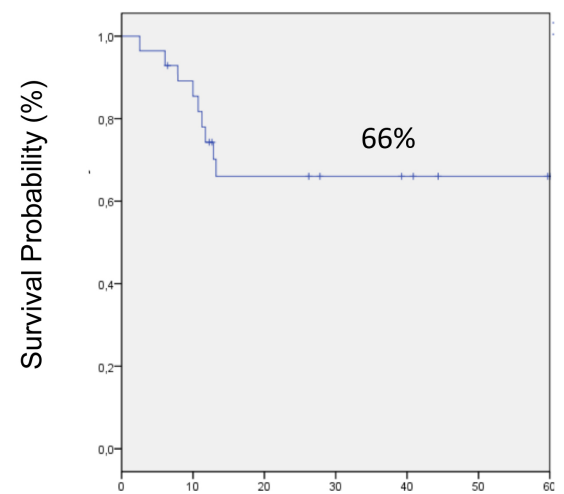

B. Event-free Survival

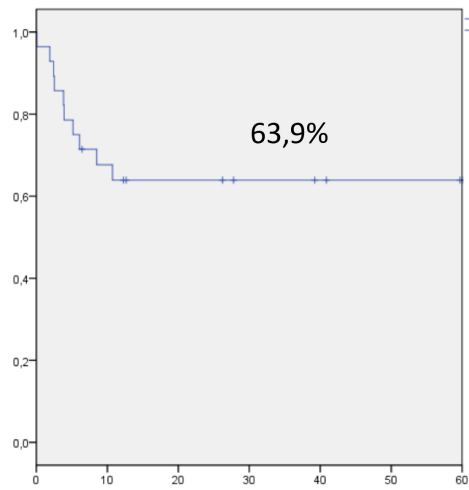

Time After Transplant (Months) 
Figure 2

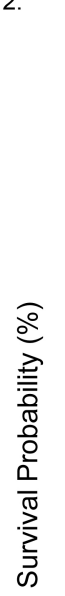

A.

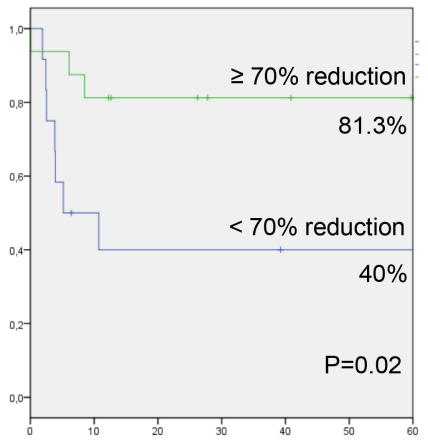

c.

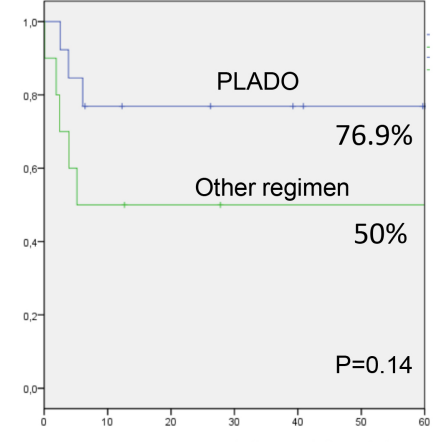

B.

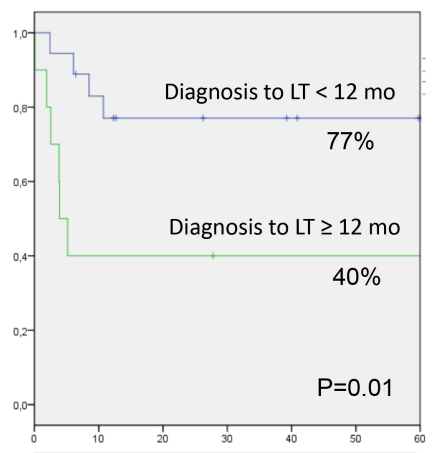

D.

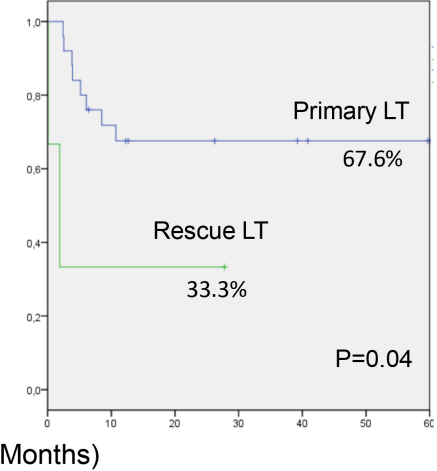

Figure 3.

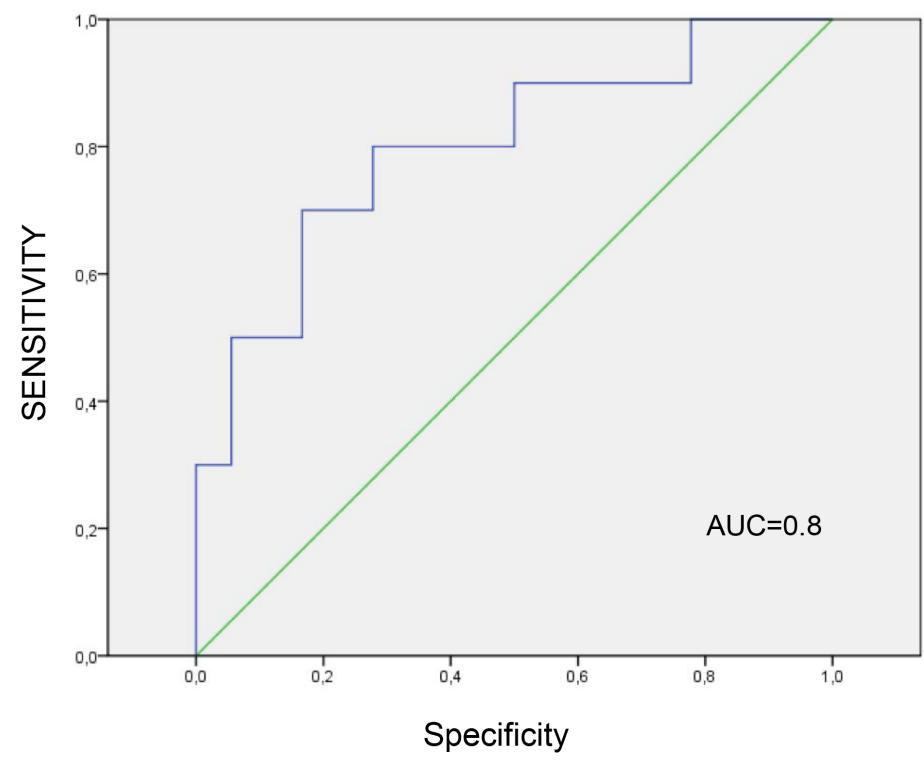


Figure 4.

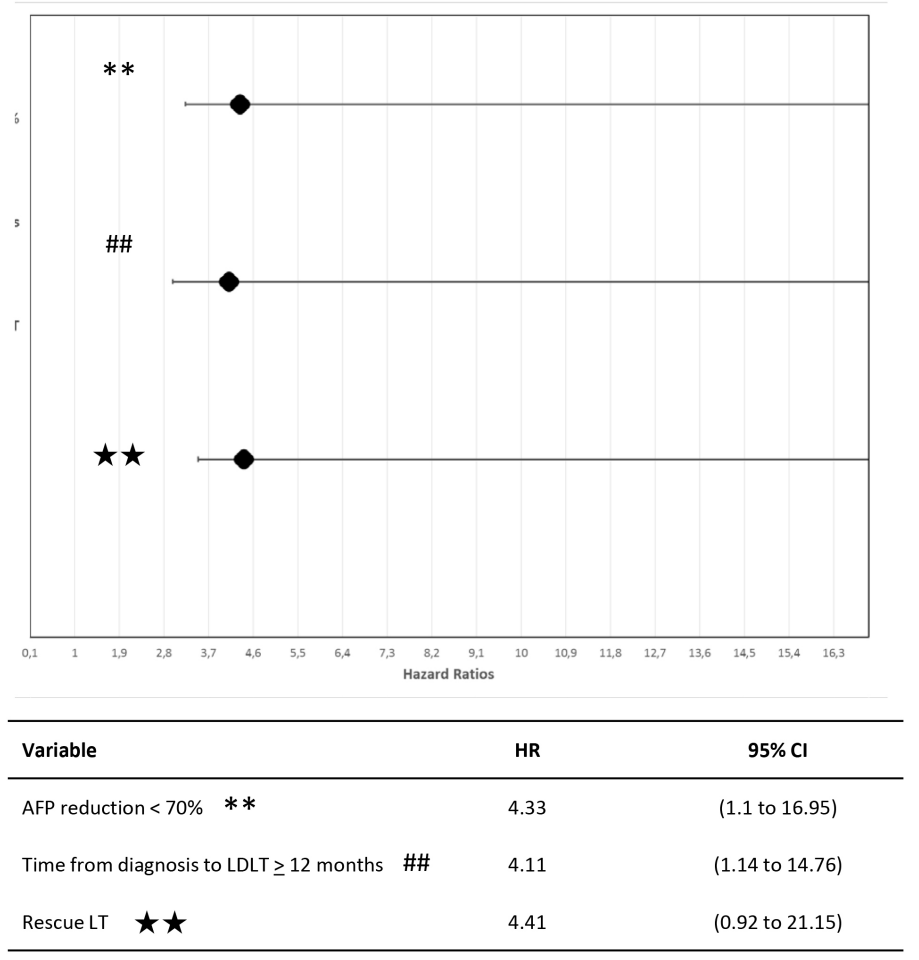

Figure 5 .

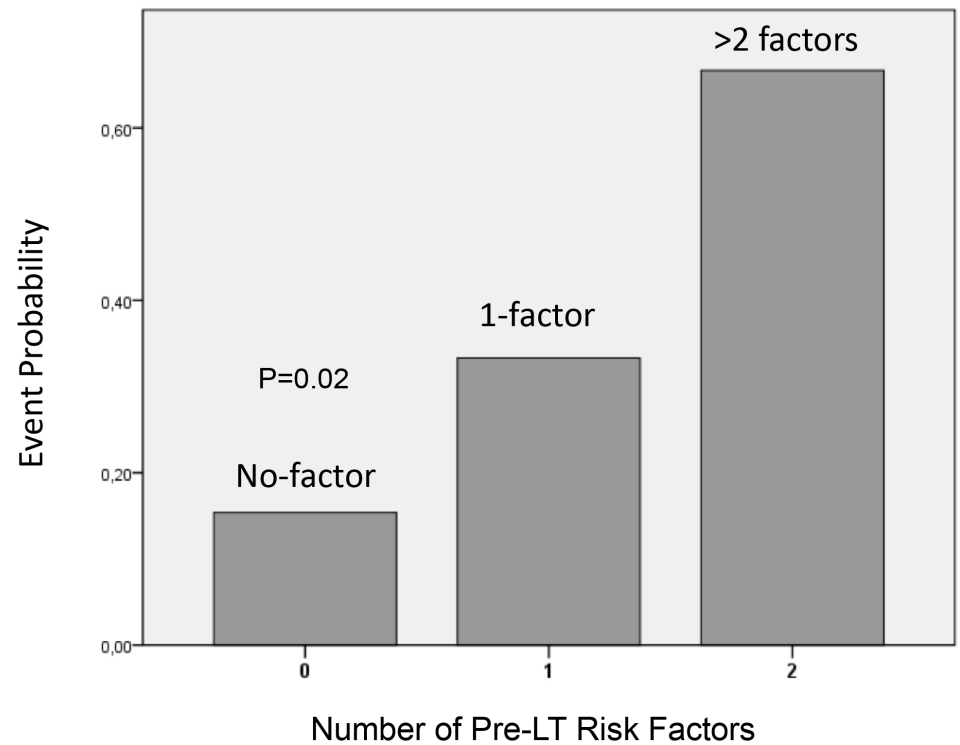

Hosted file

Table 1HBV3.pdf available at https://authorea.com/users/403341/articles/514842-unresectable- 
hepatoblastoma-living-donation-and-pre-transplant-factors-associated-with-event-freesurvival 